\title{
Coactivation and comprehension: Contribution of text variables to the illusion of knowing
}

\author{
WILLIAM EPSTEIN, ARTHUR M. GLENBERG, and MARGARET M. BRADLEY \\ University of Wisconsin, Madison, Wisconsin
}

\begin{abstract}
When a reader's self-assessment of comprehension is high, but an objective assessment reveals that comprehension is faulty, an illusion of knowing has occurred. This illusion is observed when subjects who have been instructed to find contradictions between sentences in an otherwise coherent text fail to do so, but claim comprehension of the text. Three variables intended to modulate the frequency of contradiction detection and hence the illusion of knowing were manipulated. Illusions of knowing were more frequent when the contradictions involved inferences, when the contradictory sentences were in separate paragraphs, and when contradictory sentences used paraphrase to convey the same concepts. These effects are related to an activation account of contradiction detection.
\end{abstract}

Discrepancies between self-assessed and objectively assessed levels of understanding are not uncommon. When the discrepancy involves subjective overassessment of understanding, we designate the mismatch as an illusion of knowing (Glenberg, Wilkinson, \& Epstein, 1982). When it occurs, the illusion is likely to retard learning. A student who overassesses understanding may be inclined to redeploy processing resources from the requirements of text comprehension. In addition, in expository texts that exhibit an orderly logical progression of arguments, such as texts designed to instruct in science, early failure will cascade through the ensuing text.

The current study was designed to identify one of the factors that determine the occurrence of the illusion of knowing during reading of expository text in science. In order to examine the illusion under controlled conditions, we turned to a procedure that we had employed successfully in our earlier (Glenberg et al., 1982) investigations of the illusion. The contradiction procedure presents texts for study under instructions to read as if in preparation for examination. The reader is informed that

This research was funded in part by a grant from the Spencer Foundation to the School of Education of the University of Wisconsin, Madison, and by National Institute of Education Grant NIE-G-81-0009 to the Wisconsin Center for Education Research. Any opinions, findings, and conclusions or recommendations expressed in this publication are those of the authors and do not necessarily reflect the views of the National Institute of Education or the Department of Education. We thank Leroy Lee and members of the science faculty of James Madison Memorial High School for their assistance and cooperation. We also thank Nancy Cauthen and Naomi Swanson for assisting in the preparation of the texts and in data collection. Requests for reprints may be sent to the authors at the Psychology Department, University of Wisconsin, 1202 West Johnson Street, Madison, Wisconsin 53706. a number of the texts may incorporate one or more contradictions, that is, assertions that are not consistent with the preceding text. If a contradiction is encoun. tered, the task is to designate the contradicting statement and the contradicted statement. At the conclusion of the text, understanding of the text is rated, and the subject then completes a brief objective test over the text. The event we will call an illusion of knowing is defined by the joint occurrence of failure to detect a contradiction and a positive self-assessment (rating) of understanding.

The key assumption underlying use of the contradiction procedure is that failure to detect a contradiction is a valid index of comprehension failure. Other indices are possible, for example, performance on an objective test, recall measures (including summarization), and the ability to draw inferences from the material. On at least three grounds, contradiction detection is preferable. First, contradiction detection provides a measure of comprehension while the subject is reading. Many measures are confounded with memory for the material tested after reading. Second, failure to detect a contradiction is scored easily. Scoring does not have to be corrected for guessing and does not rely on a particular theoretical analysis of text. Finally, ability to detect a contradiction is very close to what we normally mean by comprehension. Authors intend readers to understand main ideas presented in texts. If, however, a reader cannot detect a contradiction of a main idea (inserted into the text by an experimenter), then it is unlikely that the reader has understood the text as the author intended. This assertion has particular force in the case of scientific texts. The macrostructure of exposition in science typically is a tight network of propositions. Although a conscientious reader who does not achieve comprehension may acquire information at the level of 
the text microstructure, the failure to detect a fundamental contradiction implies strongly that the macrostructure, that is, the relationships among propositions, is represented inaccurately or incompletely.

Why do illusions of knowing occur? The complete answer to this question must offer an explanation for both features of the event: failure to detect the contradiction and overassessment of understanding. Here we are concerned only with developing an explanation of the failure to detect. One approach to this task is to set out the necessary conditions for detection of contradiction and then look to those conditions for the explanation of the failure to detect. One necessary condition for detection of contradiction is concurrent activation of the memory representations of the contradicting ideas. Although concurrent activation may not be sufficient to ensure detection, failure of coactivation of the constituents guarantees failure to detect the contradiction. The specific aim of this study was to examine the hypothesis that failure of coactivation is one of the determinants of the failure to detect contradictions in the illusionof-knowing paradigm.

The general plan of the experiment was to introduce a number of variables that appear in naturally occurring texts and that reasonably can be expected to affect the likelihood of coactivation. The coactivation hypothesis would be supported if the frequency of illusions of knowing was higher under those conditions that were less favorable to coactivation. The variables we chose to examine were proximity of the constituents, identity of wordings of the constituents, and whether the contradiction was explicit or implicit, that is, whether the contradiction was evident in direct comparison of statements or was one that involved comparison with an unwritten inference.

We assumed that proximity of the constituents favors coactivation. This assumption is so widely held among diverse theorists that special justification is not required. The effect of proximity was tested by varying the distance in the text between the contradicted and contradicting statements. In a comparison between a condi- tion that presented the critical constituents in the same paragraph and a condition that presented the constituents in different paragraphs, coactivation would be less likely under the latter condition and the number of illusions would be greater.

The manipulation of identity of wording compared a verbatim condition and a paraphrasal condition. The former condition employed identical wording to express the same constituent ideas when they appeared in the critical statements. The latter condition used a paraphrase to express the linked constituent ideas. These conditions are illustrated in the bottom of Table 1. The letters $a, b$, and $c$ represent constituent ideas; $R$ represents a relation connecting two constituents. Examples of constituents and relations are given in the top of Table 1. In the verbatim condition, a constituent idea was expressed by identical wording wherever that idea occurred in the text. As an example, consider the verbatim-explicit condition. The contradicting ideas are presented in sentences $a R c$ and $a R c$ (the bar over the $R$ indicates negation of the relation); the wordings of the constituent ideas a and $c$ are the same in both sentences. In the paraphrasal conditions, wordings of the constituent ideas changed across sentences (indicated by a prime). Thus, in the paraphrase-explicit condition, the contradictory ideas presented in sentences $(\mathrm{aRc})^{\prime}$ and $\mathrm{a} \overline{\mathrm{R}} \mathrm{c}$ were conveyed by different wordings of the constituents. We assumed that coactivation would be less likely in the paraphrasal condition and that, as a result, illusions of knowing would be more frequent in the paraphrasal condition.

Related effects of proximity and identity of wording have been reported by Cirillo (1981) and Hayes-Roth and Thorndyke (1979). In his study of story comprehension, Cirillo examined the hypothesis that a sentence should be easier to comprehend and require less time to read if it is coreferential, for example, shares a noun phrase with an earlier sentence. However, coreference will confer an advantage only if the referents are coactive. Cirillo assumed that the probability of coactivation is favored by proximity of the coreferents. To test

Table 1

Critical Statements for One Base Text and the Organization of Statements to Form Conditions

$\mathrm{aRb} \quad$ (a) Plants - (R) have - (b) the ability to manufacture all necessary vitamins.

bRc (b) The ability to manufacture all necessary vitamins - (R) is - (c) a strong protection against extinction.

$(b R c)^{\prime} \quad$ (b') Synthesis of needed nutrients - (R) is - (c) a mechanism which virtually guarantees survival of a species.

aRc (a) Plants - (r) have - (c) a strong protection against extinction.

$(\mathrm{aRc})^{\prime} \quad\left(\mathrm{a}^{\prime}\right)$ Trees and other forms of vegetation - (R) have developed - ( $\left.\mathrm{c}^{\prime}\right)$ abilities which practically guarantee survival of the species.

$\mathrm{a} \overline{\mathrm{R}} \mathrm{c} \quad$ (a) Plants $-(\overline{\mathrm{R}})$ have no - (c) strong protection against extinction.

\begin{tabular}{|c|c|c|c|c|}
\hline \multicolumn{2}{|c|}{ Verbatim } & \multicolumn{2}{|c|}{ Paraphrase } & \multirow[b]{2}{*}{ Control } \\
\hline Explicit & Implicit & Explicit & Implicit & \\
\hline $\begin{array}{l}\mathrm{aRb} \\
\mathrm{aRc} \\
\mathrm{a} \bar{R} \mathrm{c}\end{array}$ & $\begin{array}{l}\mathrm{aRb} \\
\mathrm{bRc} \\
\mathrm{a} \bar{R} \mathrm{c}\end{array}$ & $\begin{array}{l}\mathrm{aRb} \\
(\mathrm{aRc})^{\prime} \\
\mathrm{a} \overline{\mathrm{R}} \mathrm{c}\end{array}$ & $\begin{array}{l}\mathrm{aRb} \\
(\mathrm{bRc})^{\prime} \\
\mathrm{a} \overline{\mathrm{R}} \mathrm{c}\end{array}$ & $\begin{array}{l}\mathrm{aRb} \\
\mathrm{bRc} \\
\mathrm{aRc}\end{array}$ \\
\hline
\end{tabular}


this assumption, the target sentence and its critical antecedent sentence were either immediately contiguous in the text or were separated by three intervening sentences. Under Cirillo's Condition Mn, which closely resembled the reading conditions of the present study, Cirillo found that the target sentence was read faster when its coreferent was an immediate precursor than when its coreferent was separated from the target. Hayes-Roth and Thorndyke studied integration of facts acquired from narrative texts. They found that knowledge integration as assessed by a variety of measures was facilitated by proximity of related facts and by identical rather than paraphrased wordings of the related facts. Hayes-Roth and Thorndyke's interpretation of these findings relies heavily on assumptions concerning activation similar to those we have advanced.

It may appear that, in the interest of advancing the foregoing analysis, we have taken it as given that the representation of text in memory is exclusively a reproduction of the surface properties of the text: What is proximate in the text is proximate in the representation of the text, and the content and relationships in the text are mirrored directly in the representation. In fact, it is not necessary to subscribe to this strong view in order to give credence to our treatment of proximity and identity of wording. A more defensible position will suffice to accommodate our analysis. According to this view, representation of text in memory comprises two types of rendering: One recapitulates the nominal text (that is, the representation preserves the lexical content and its temporal or spatial arrangement); the other represents the meaning and deep structure of the text, perhaps as an organized network of propositions (Anderson \& Paulson, 1977; Keenan \& Kintsch, 1974).

The notion of dual representation may be used to justify the third experimental manipulation, which compares explicit and implicit contradictions. In the explicit condition, the second critical statement, $\mathrm{aR} c$, was explicitly contradicted by the conclusion, $\mathrm{a} \overline{\mathrm{R}} \mathrm{c}$. (See Table1.) In the implicit condition, there was no direct inconsistency between the second and third statements. Instead, the contradiction involved an inference encouraged by the linkage of the first statement, $\mathrm{aRb}$, and the second statement, bRc. The third statement, a $\bar{R} c$, contradicted the putative but unstated inference, aRc. We assumed that coactivation is less likely in the implicit than in the explicit condition and that, on this account, illusions of knowing will be more frequent in the implicit condition. The reasoning underlying this expectation rests on the notion of dual representation. Whereas both the surface form and the deep (propositional) form of the explicit version of the contradicted statement are represented, only the propositional form of the implicit version is represented. Text that is available in only a single representation is less likely to be activated than text available in dual representation. A set of studies by Keenan and Kintsch
(1974) supported this reasoning. They found that reaction times to verify explicitly presented sentences were significantly faster than reaction times for inferences never presented in the text even though independent evidence had shown that the inferences were highly probable. Verification time frequently has been used to assess activation, and the difference in verification time in these studies is taken by Kintsch (1977, chap. 6) as evidence that the dual representation of explicit statements confers readier activation than does the single representation of inferred statements.

In summary, we proposed to examine the hypothesis that one determinant of failure to detect a contradiction is failure of coactivation of the critical related statements. Drawing on widely held assumptions concerning activation as well as on selected experimentation, we contended that coactivation would be less likely for two statements far apart in the text, less likely when identical wordings were not used in the linked statements, and less likely when the contradicted statement was inferred. These three conditions should exhibit more frequent illusions of knowing than their respective comparison conditions.

\section{METHOD}

\section{Subjects}

The 205 subjects were students enrolled in chemistry classes at a middle- to upper middle-class high school. In Semester 1, we collected data from 133 students in their chemistry classes (six classes of approximately 22 students). The following semester, we collected data from 72 students after normal school hours. These students participated in small groups (6 to 12 students per group) and were paid $\$ 4.00$ for their participation.

\section{Materials}

Three topics, the origin of the solar system, physiological mechanisms that control eating, and vitamins and evolution, were selected from various high school science texts. For each topic, a base text was created by editing and rewriting the information presented in the textbooks. These base texts were four to six paragraphs ( 369 to 504 words) and were relatively self-contained. Three high school science teachers judged the reading level of the base texts to be appropriate for high school students.

Six critical statements were written for each base text. Examples are given in Table 1 . The $\mathrm{aRb}$ sentence related $(\mathrm{R})$ one concept (a) to another (b). The bRc sentence introduced a third concept (c). Repetition of the symbol $b$ indicates that the same words were used to convey the concept. The sentence (bRc)' provided the same information as $b \mathrm{Rc}$, but with different content words (indicated by the prime). Two other sentences, aRc and $(\mathrm{aRc})^{\prime}$, were logical consequences of the conjunction of $a R b$ and either $b R c$ or $(b R c)^{\prime}$. The sixth critical statement was $a \bar{R} c$, the negation or contradiction of aRc.

Experimental passages (those containing contradictions) were constructed by inserting three critical sentences into a base text. The first, $a R b$, and the last, $a \bar{R} c$, were always the same. The middle sentence for the explicit-verbatim texts was aRc. This sentence is explicitly contradicted by $\mathbf{a} \bar{R} c$, the third sentence. Also, except for the negation, the contradiction is a verbatim repetition of the contradicted sentence. The explicitparaphrase texts used $(a \bar{R} c)^{\prime}$ as the middle sentence. Again, the third (contradicting) sentence, $a \bar{R} c$, contradicts a statement 
explicit in the text; however, the two sentences use different content words.

The implicit-verbatim texts included $a R b, b R c$, and $a \bar{R} c$. Sentence $a \bar{R} c$ contradicts a sentence derivable from the first two critical sentences, but not included explicitly in the text. The implicit-paraphrase texts included $\mathrm{aRb},(\mathrm{bRc})^{\prime}$, and $\mathrm{a} \overline{\mathrm{R}} \mathrm{c}$.

Control texts did not contain contradictions. These texts contained sentences $\mathrm{aRb}, \mathrm{bRc}$, and $\mathrm{aRc}$.

A final text variable was the proximity of the three critical sentences in the text. The within-paragraph condition incorporated the three sentences into a single paragraph composed of just those three sentences. This paragraph was inserted into the base text at a location that maintained the organization and flow of the text, but never prior to the third paragraph in the base text and never after the last paragraph in the base text. For the between-paragraph condition, each of the three critical sentences was incorporated into a separate paragraph of the base text. The first paragraph was never used. Incorporation of these critical sentences required minor changes (such as adding or deleting a conjunction) in the base texts.

For each subject, three texts and a response sheet for each text were arranged in a booklet such that the text occurred on the left side of the booklet and the response sheet on the right side of the booklet. In this way, both the text and the response sheet were available to the subject during reading of the text. All sentences in each text were numbered for easy identification. The response sheet consisted of two parts. At the top of the sheet, a short set of instructions indicated that, if a contradiction was found in the text, the numbers of the contradictory statements, as well as a short explanation of what the contradiction was, should be written in the space provided. The bottom part of the response sheet had a scale that subjects were to use to rate their understanding of the text. The scale ran from 1 to 4 , with 1 indicating little comprehension and little expectation of doing well on a test over the content of the text, and 4 indicating good comprehension and an expectation of answering all the test questions correctly. On the back of the response sheet were three multiple-choice questions testing for information in the text. In addition, the booklet contained a practice passage and a response sheet preceding the three texts, and a postexperimental questionnaire that followed all texts.

\section{Design}

The experiment conformed to a factorial combination of three independent variables, type of contradiction (explicit or implicit), wording of the constituents of the contradiction (verbatim or paraphrase), and proximity of the constituents (within paragraph or between paragraph). The type of contradiction was manipulated between subjects. The other two variables were manipulated both within and between subjects according to the following scheme: Each subject read three texts (one on each of the three topics). One text did not contain a contradiction (control). One text contained a verbatim contradiction that was either within or between paragraphs. The other text contained a paraphrase contradition in the other proximity condition. Within pairs of subjects, the four texts containing contradictions corresponded to the four cells of a 2 (wording) $x$ 2 (proximity) design. Extraneous variables, such as the order of the three topics, the order of the experimental conditions, and the nature of the control text (critical sentences presented within or between paragraphs), were held constant within each pair of subjects. All combinations of these extraneous variables were sampled approximately equally often across pairs of subjects.

\section{Procedure}

Prior to the experiment, parental consent was secured for participation of the students.

The subjects were told that the experiment was designed to investigate comprehension of written text. They were instructed to read each passage carefully, as if they were studying for a test, and that three multiple-choice questions would test their understanding of the material. In addition, the subjects were instructed to look for parts in the texts that did not make sense. Examples of implicit and explicit contradictions were presented in the instructions, along with an explanation of the nature of the contradiction. The subjects were encouraged to ask questions.

The subjects were instructed to write the numbers of the contradictory statements and a short explanation of why these sentences were contradictory. They were also instructed how to use the 4-point response scale at the bottom of the response sheet after reading each passage, and then to turn the page and answer the three multiple-choice questions, without looking back at the passage.

The practice passage was then read aloud by the experimenter, and the contradictory information in that passage was noted and written down on the response sheet by each subject. The subjects rated their understanding of the practice passage and answered the test questions. Following the practice passage, the subjects proceeded through the booklets at their own rate. After all passages had been read, the subjects filled out a postexperimental questionnaire that queried several aspects of the experiment.

\section{RESULTS}

The data from 11 subjects who participated in Semester 1 were eliminated from the analyses. The data from 5 subjects were eliminated because the subjects did not follow instructions $(n=3)$, did not receive the correct passage $(n=1)$, or left the experiment before its completion $(n=1)$. Also eliminated were data from subjects paired with these 5 . Finally, 1 subject who did not have a partner was eliminated. The final data set included 194 subjects, 122 from Semester 1 and 72 from Semester 2. The explicit-contradiction condition con tained 98 subjects, and the implicit-contradiction con dition contained 96 subjects.

An illusion of knowing was scored if a contradictory statement was not indicated on the response sheet and the subject rated understanding of the passage as high ( 3 or 4 on the response scale). The proportions of illu sions of knowing in the various conditions are pre sented in Table 2.

The analysis of variance of these data was conducter with a hierarchical multiple regression procedure (Coher \& Cohen, 1975) using Model II error terms. The unit o analysis was pairs of subjects. The between-subject variables were semester in which the data were collecter (entered first), type of contradiction, and their inter action. Only the type of contradiction was significan

Table 2

Proportions of Illusions of Knowing

\begin{tabular}{lccccc} 
& \multicolumn{2}{c}{ Explicit } & & \multicolumn{2}{c}{ Implicit } \\
\cline { 3 - 5 } Wording & Between & Within & Between & Within \\
\hline Paraphrase & .29 & .16 & .47 & .30 \\
Verbatim & .22 & .14 & .19 & .17 \\
\hline
\end{tabular}

Note- "Between" and "Within" refer to proximity. 
$[F(1,23)=8.98]$. The proportions of illusions of knowing were .20 for the explicit condition and .28 for the implicit condition. The direction of this difference is what is expected by the activation account. Compared with explicit contradictions, implicit contradictions are difficult to detect because (1) the reader must infer the statement that is later contradicted, and (2) this inference is difficult to activate because it lacks a lexical component in the representation of the text.

For the within-subjects hierarchical analysis, variance associated with the base text (topic) was removed before the effects of the other variables were assessed. Two significant main effects were found. For wording, the proportions of illusions of knowing were .18 in the verbatim condition and .30 in the paraphrase condition $[F(1,277)=3.94]$. For proximity, the proportions of illusions of knowing were .19 in the within-paragraph condition and .29 in the between-paragraph condition $[F(1,277)=10.94]$. Both of these effects are consistent with the activation account of contradiction detection." Although the data in Table 2 hint at interactions between wording and proximity and between wording and type of contradiction, none of the interactions involving these three variables had $\mathrm{F}$ ratios greater than 1.0.

The pattern of main effects was fairly consistent across the three topics. Two of the topics generated patterns of main effects consistent with the three main effects for type of contradiction, wording, and proximity. The third topic generated the wording and proximity patterns; however, it was associated with a small reversal of the type-of-contradiction effect. For this topic, the relative frequencies of illusions of knowing were .30 for the explicit condition and .26 for the implicit condition.

The data from the control passages were scored for false alarms. A false alarm was scored when subjects indicated a contradiction involving one or more of the critical sentences ( $a R b, b R c$, and $a R c)$, which, in the control passages, did not form contradictions. The average false-alarm rate was .20 . A false alarm measures, in some sense, a bias to indicate contradictions. A bias of this sort would reduce the likelihood of observing illusions of knowing because illusions of knowing depend on the failure to indicate contradictions. Seen in this perspective, even the smaller of the proportions in Table 2 are impressive.

The single variable manipulated for the control passages was proximity of the critical sentences. The false-alarm rates were .16 for the between-paragraph condition and .24 for the within-paragraph condition. The difference was due solely to one topic for which the within-paragraph false-alarm rate was unusually high, .38. When this topic was deleted from the analysis, the between-paragraph and within-paragraph false-alarm rates were both .16. When data from this topic were deleted from the illusion-of-knowing analysis, the proportion of illusions of knowing for the betweenand within-paragraph conditions $(.34$ and .18 , respec- tively) did not change appreciably from the overall proportions (.29 and .19 , respectively).

\section{DISCUSSION}

Why do readers who have been alerted to the presence of contradictions and who have been motivated to read for comprehension fail to detect a significant proportion of contradictions? As a partial answer, we suggested that one contributing factor is failure of coactivation of the contradicting and contradicted information. To test this hypothesis, we examined the effect of frequency of detection failures of three variables presumed to influence the likelihood of coactivation. The results were compatible with our hypothesis. Variations of proximity, identity of wording, and type of inference, which were presumed to influence coactivation, had correlated effects on the frequency of detection failures.

Although this study has emphasized failure of coactivation, we have also alluded to the fact that coactivation is not sufficient to guarantee detection of contradiction. A striking demonstration of the insufficiency of coactivation is provided in Markman's (1979) studies of elementary school children's awareness of inconsistencies in texts. In her second study, Markman ensured concurrent activation of the inconsistent sentences by having the children repeat the incompatible sentences together. Despite concurrent activation, third-grade children failed to detect the incompatibility and treated the text as comprehensible.

Detection of contradiction depends on the application of tests to the coactivated representations. The tests are not automatic and are not applied unfailingly at the appropriate level (Glenberg et al., 1982). Finally, we should note that successful detection of contradiction does not confer absolute immunity from the illusion of knowing. Having detected an inconsistency, a reader may restructure the interpretation of earlier presented knowledge so that coherence is restored. With the restoration of coherence, the reader may pronounce satisfaction in the level of understanding achieved. Evidence of the deployment of "fix-up strategies" of this sort was presented in an experiment by Mosenthal (1979). In the experiment reported here, however, it is unlikely that processing of a contradicting sentence triggered the sort of knowledge restructuring that would mask contradictions. The subjects were explicitly instructed to report contradictions. Thus, unless contradiction detection and subsequent knowledge restructuring are opaque to conscious processing, this explicit instruction ensures that subjects will report contradictions, rather than restructure knowledge. Indeed, the not unsubstantial false-alarm rate is evidence that the subjects were attempting to detect and report contradictions, even to the extent of claiming contradictions between sentences that we judged to be noncontradictory.

The foregoing makes clear that the study reported here represents only a modest advance toward the under- 
standing of illusions of knowing in the reading of scientific text. Nonetheless, the results may have an implication for the organization of instructional text. We found that presentation of related idea units at widely separated locations in the text, departures from identical wordings in expressing the same ideas, and the use of unexpressed inferences are not conducive to coactivation. Despite these reasons for avoiding these practices in the composition of text, other considerations, substantive and stylistic, may encourage their use. One prescription for counteracting the detrimental effects of these practices is implied by the results of the present study. Introduction of intermittent concise summarizations that rehearse the main argument in a set of contiguous explicit propositions expressed in shared language may ensure that coactivation of the related ideas occurs. In turn, coactivation is an opportunity for the construction of a coherent knowledge structure.

\section{REFERENCES}

Anderson, J. R., \& Paulson, R. (1977). Representation and retention of verbatim information. Journal of Verbal Learning and Verbal Behavior, 16, 439-452.

Cirillo, R. K. (1981). Referential coherence and text structure in story comprehension. Journal of Verbal Learning and Verbal Behavior, 20, 358-367.
Cohen, J., \& Cohen, P. (1975). Applied multiple regression/ correlation analysis for the behavioral sciences. Hillsdale, $\mathrm{NJ}$ : Erlbaum.

Glenbera, A. M., Wilkinson, A. C., \& Epstein, W. (1982). The illusion of knowing: Failure in the self-assessment of comprehension. Memory \& Cognition, 10, 597-602.

Hayes-Roth, B., \& Thorndyke, P. W. (1979). Integration of knowledge from text. Journal of Verbal Learning and Verbal Behavior, 18, 91-108.

Kennan, J. M., \& Kint8ch, W. (1974). The identification of explicitly and implicitly presented information. In W. Kintsch (Ed.), The representation of meaning in memory. Hillsdale, $\mathrm{NJ}$ : Academic Press.

KInTsCH, W. (1977). Memory and cognition. New York: Wiley.

Markman, E. M. (1979). Realizing that you don't understand: Elementary school children's awareness of inconsistencies. Child Development, 50, 643-655.

Mosenthal, P. (1979). Children's strategy preference for resolving contradictory story information under two social conditions. Journal of Experimental Child Psychology, 28, 323-343.

\section{NOTE}

1. A separate analysis was run on the proportion of correct detections of contradictions, disregarding the comprehension ratings. The three variables, type of contradiction, wording, and proximity, were also significant in this analysis.

(Manuscript received July 31, 1983; revision accepted for publication February 23, 1984.) 